\title{
Use of Baidu Index to Track Chinese Online Behavior and Interest in Kidney Stones
}

This article was published in the following Dove Press journal:

Risk Management and Healthcare Policy

\section{Tengteng Wang \\ Qinghua Xia \\ Xiude Chen \\ Xunbo Jin}

Department of Urology, Shandong Provincial Hospital Affiliated to Shandong University, Jinan, Shandong, People's Republic of China
Correspondence: Xiude Chen Department of Urology, Shandong Provincial Hospital Affiliated to Shandong University, Jinan, Shandong 2500 I4,

People's Republic of China

Tel +86 I5I68863866

Email scistone_2019@163.com
Background and Aim: Baidu, currently the top online search tool in China, has developed an internet search trends gathering and analysis tool called the Baidu Index. The aim of this study was to explore the value of the Baidu Index for monitoring online kidney stonesrelated information-seeking behavior and understanding of population features and priorities in China.

Materials and Methods: The Baidu Index was queried using the term "kidney stones" for the period 2014-2018. The search volume was recorded as well as a demand graph, with a geographic and demographic distribution. For surgical treatment modalities, the medical term "extracorporeal shock wave lithotripsy (ESWL)" was analyzed and then compared to the term "kidney stone surgery" to assess changes.

Results: The search trend for the term "kidney stones" remained sustained growth over time. Searches were mostly presented by the age $20-49$, in males in the south of China. The demand graph indicated that the diagnosis, followed by the treatment, and dietary control of kidney stones had received the most attention. The search volume related to ESWL showed a similar increasing trend, but was searched with a greater volume than the term "kidney stone surgery" for most of the last 5 years.

Conclusion: The Baidu Index is a useful tool to track online health information-seeking behavior of internet users in China. The geographic and demographic characteristics in kidney stones seem to be reflected in online search trend data to some extent.

Keywords: search engine, Baidu Index, kidney stones, kidney stone surgery, extracorporeal shock wave lithotripsy

\section{Introduction}

In China, nephrolithiasis is a common urologic disorder with increased prevalence and recurrence. The 2017 study by Zeng et al $^{1}$ demonstrated that renal calculi affects approximately one in 17 adults $(6.5 \%$ of men and $5.1 \%$ of women, overall prevalence rate was $5.8 \%$, ) currently in China. Considering kidney stone disease was a healthcare and socioeconomic burden in China, it had spurred additional research interest in intervention and prevention of kidney stones. ${ }^{2,3}$

The development of the internet has greatly changed people's lives, especially in that the expansion of search engines has further enhanced the value of the internet as a tool for life and work. Referring to the 44th China Statistical Report on Internet Development, China had 854 million netizens, $81.3 \%$ of whom used search engines to obtain information. It was estimated that $70.5 \%$ of netizens used the search engine to seek health information. With Google's 2010 withdrawal, Baidu was currently the top search tool in mainland China. About $90.9 \%$ of internet users 
ranked Baidu as their preferred and dominant search tool. In 2018, over 10 billion searches were conducted daily by Baidu in China., ${ }^{4,5}$ Analysis of these online search trend data can represent patterns of health information-seeking behavior and interest of internet users on population level.

Internet search trends gathering and analysis tools, like the Baidu Index, were developed to provide a new resource of information for understanding public discourse, epidemiology, and features of various diseases, including kidney stones. To our knowledge there has been no research that focused on Chinese online discourse in kidney stones. We sought to explore whether the interest search trend data reflects actual kidney stones burden and to find out the features and priorities of kidney stone patients. We sought to explore the value of internet search data for monitoring online kidney stones-related information-seeking behavior and to find out the features and priorities of kidney stones patients.

\section{Materials and Methods}

The Baidu Index is a big data sharing platform constructed by Baidu's massive users' behavior information. Here, you can find out the search trends of selected keywords, gain insight into the changes of netizens' demand, monitor the trends of media public opinion, and locate user characteristics. The Baidu Index is updated daily, providing a PC search index at any time since June 2006 and a mobile wireless search index since January 2011. The Baidu Index represents a normalized search volume for selected keywords, instead of the absolute search volume. Other than that, each online query returns a demand graph, a demographic and geographic distribution. Up to five keywords can be simultaneously compared on a single or multiple charts. More details about the Baidu Index are shown at http://index.baidu.com/Helper/?tpl=helpandword=\#pdesc.

To examine the internet users' attention to kidney stones, we queried the Baidu Index (http://index.baidu.com) using the Chinese search term "kidney stones" within mainland China from January 1, 2014 through December 31, 2018. For the term "kidney stones" online search trend data was gathered. For concrete surgical treatment procedures, the medical term "extracorporeal shock wave lithotripsy (ESWL)" was analyzed. This search queries were then compared through the Baidu Index to the terminology "kidney stone surgery". Related search trend data were collected and then studied to evaluate changes of population interest in search queries over time. "Percutaneous nephrolithotomy" and "ureteroscopy lithotripsy" were not included in the Baidu Index keywords database due to the low search volume.

\section{Results}

We found that the online search trend for the term "kidney stones" remained sustained growth during the 5-year period (Figure 1). Separate analysis results illustrated that the mobile search index showed an upward trend similar to the holistic Baidu Index, and conversely the personal computer (PC) search index has gradually decreased below the average line since November 22, 2016. Geographic differences of the search volume related to kidney stones was well represented by a side by side comparison of the Baidu Index-generated China map, in which bluer regions, provinces, and cities represent a greater search volume (Figure 2). A basically similar pattern was seen in these maps, reflecting the known greater kidney stone prevalence in south China. However, the maximal search queries came from more socioeconomically developed areas, where people have greater access to and use of interest and smart phones. The demographic profiles showed that "kidney stones" related search queries were mostly from the age 20-49 (83\%), male (50.18\%) groups (Figure 3).

Top-searched terms ranking by category indicated what Baidu users were interested in most was the diagnosis, followed by the treatment, and dietary control of kidney stones. Search trend data suggested the treatment of kidney stones followed by concomitant diseases had the fastest growing interest among Chinese netizens (Tables 1 and 2). The search query volume related to ESWL yielded a similar increasing trend, but was searched with a greater volume than the terminology "kidney stone surgery" for most of the last 5 years (Figure 4).

\section{Discussion}

Despite the considerable healthcare expenditure imposed on China by nephrolithiasis, China's vast geographic variations and large population make it quite difficult to evaluate the true prevalence of kidney stones and find out the characteristics and demand of individuals with renal calculi. Fortunately, along with the widespread application of internet and public growing reliance on the search engine as a surrogate for health-related information in China, the use of internet big data in healthcare field holds promise and also it may supplement and expand the current clinical and epidemiologic data. ${ }^{6}$ According to the latest statistical report, the search engine is the first-choice 


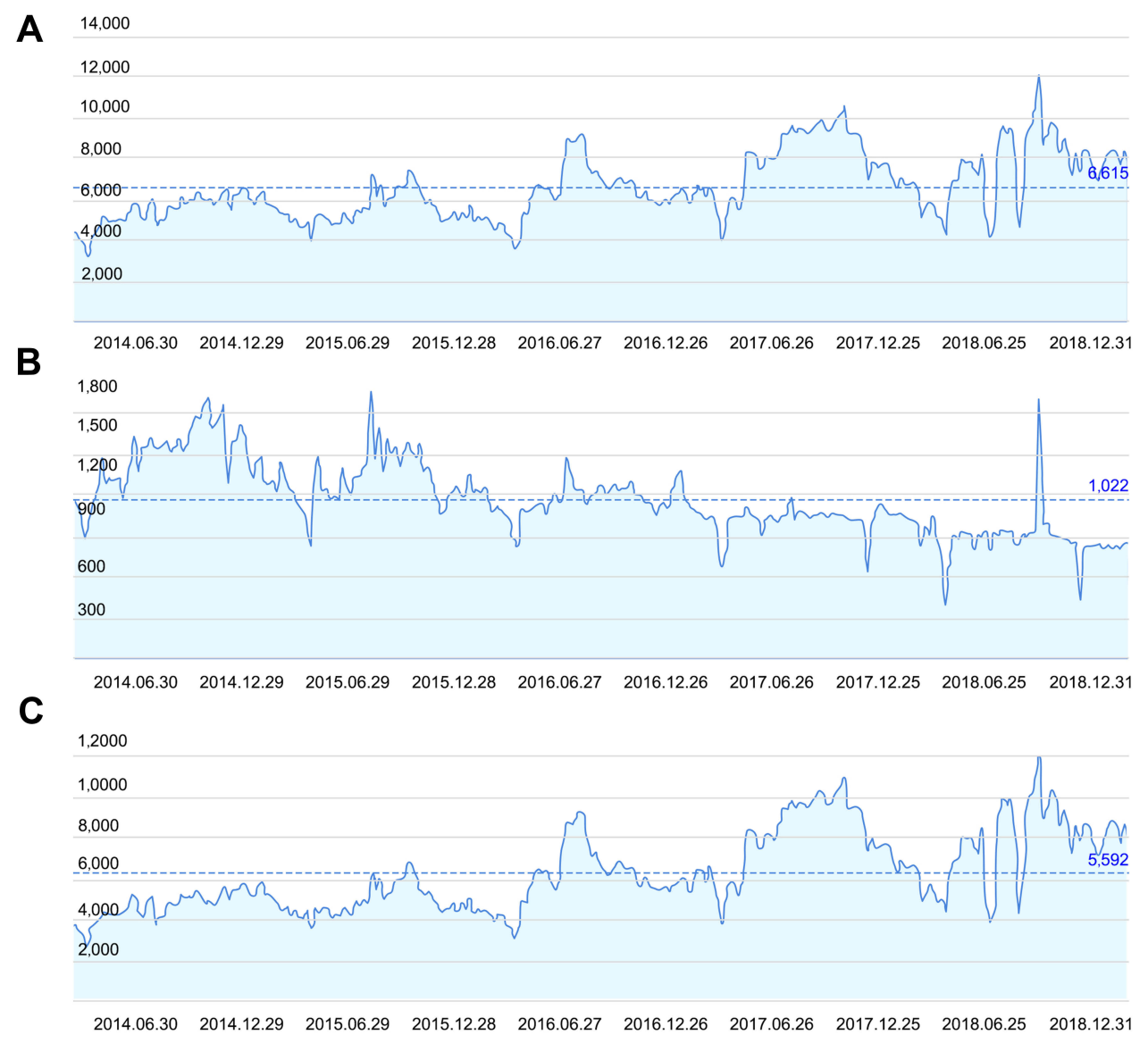

Figure I Baidu search volume index in China during 20I4-2018 for "kidney stones". The average value of search volume. (A) The overall search trend index. (B) Personal computer search index. (C) Mobile wireless search index.

channel for obtaining health related information. Behavior measures of internet users' search queries could guide communication and policy planning. ${ }^{7}$ Our study demonstrated the potential in using online search trend data to represent the real-world situation for individuals with kidney stones in China.

With Google's 2010 withdrawal from China, the Baidu Index has been mostly used to monitor public health and predict disease in China. Based on the Baidu Index, the disease prediction product developed by Baidu and the Chinese Center for Disease Control and Prevention can provide real-time data of infectious diseases such as influenza, hand-foot-mouth disease, sexually transmitted diseases, AIDS, hypertension, Diabetes, lung cancer, and breast cancer. Simultaneously, it is also used to predict the epidemic trend of diseases, acting as a powerful complement to the traditional detection system. ${ }^{8}$ Nevertheless, utilization of the Baidu Index in healthcare research is largely unexplored and massive amounts of data remain to be extracted, particularly in patients with urological diseases. Prior studies using another more popular internet search trend tool (Google trends) overseas have focused on geographic and temporal variations in urolithiasis, epidemiology of kidney stones, and population interest in surgical treatment modalities of kidney stones. ${ }^{9,11}$ There has never been a kidney stones-related study using the Baidu Index in China. Our current research represents the first attempt to explore Chinese online behavior and interest in kidney stones, confirming that Baidu Index data can gauge "epidemiology" of kidney stone disease and public interest in treatment modalities.

Our study illustrated that search volumes for the term "kidney stones" had sustained growth over the past 5 years. This might be attributed to an increase in kidney stones prevalence, a high public health awareness, or the increased internet penetration, especially with the universal availability of smart phones. In detail, the search volume spiked in the summer months annually, corresponding to longer daylight hours and warmer temperatures leading to higher incidence of renal calculi in the summer and in south China. Evidence 
A

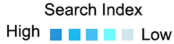

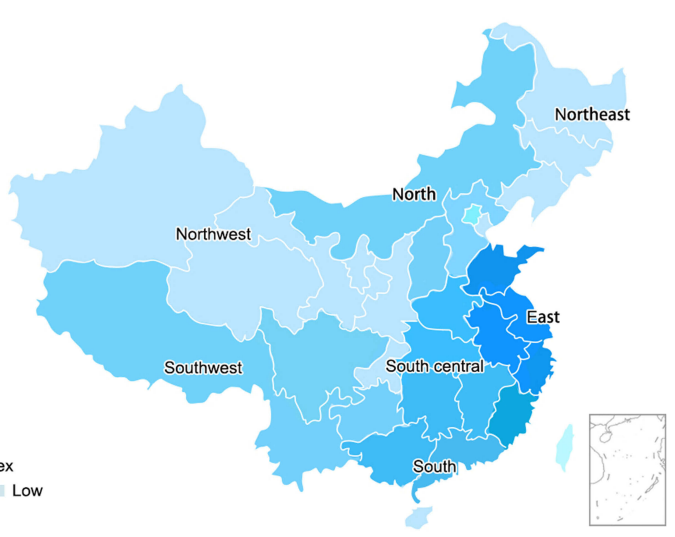

B

High

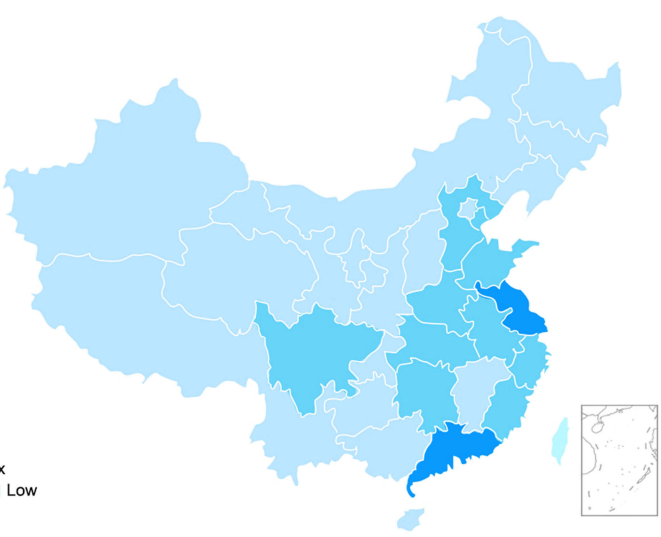

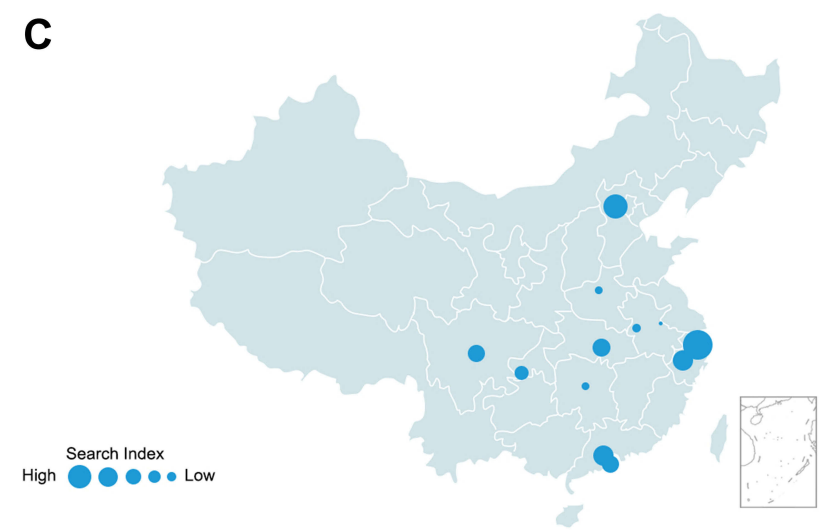

Figure 2 Baidu Index maps for "kidney stones" by region (A), province (B), and city (C), 2014-2018.

shows that prolonged sunlight exposure may increase stone formation risk via calcium absorption and vitamin D production. ${ }^{12,13}$ Likewise, exposure to high temperatures increases the risk of stone formation, probably through dehydration, acidosis, and mineral super-saturation. ${ }^{14}$ In the study, we further found that the mobile search index of renal calculi did show an upward trend and the PC search index evidenced a clear decreasing trend, possibly due to the advancement of technology on the internet and mobile devices in recent years. Given the increased use of mobile devices among netizens to access health information of kidney stones, health educators with the aim to motivate individuals to prevent stone disease through habit and behavior intervention can better guide their target audience with the help of mobilebased social media.

In the analysis of geographical differences, we found that interest search trends in the Baidu Index could reflect actual clinical trends in stone disease on a national level to some extent. The search volume of kidney stones in south China was much higher than in north China, consistent with the known geographic pattern in kidney stones incidence and prevalence, which reflected the fact that the environment difference strongly impacted kidney stone formation. To explore the regional difference, China is divided into the traditional seven geographic regions. Next, the seven regions were sorted in descending order by the volume of search queries, as shown in Figure 2 (east, south, south central, north, southwest, northeast and northwest, consistent with the social and economic development level). A large amount of variability was seen between the Baidu Index search volume and actual regional stone prevalence, according to the data from the latest stone epidemiologic study by Zeng et $\mathrm{al}^{1}$ (sorted in descending order: south, southwest, northeast, south central, east, northwest, and north). Similar to the regional distribution, the Baidu Index search volume exhibited profound differences to reported stone prevalence at the province and city levels. The reported prevalence of renal calculi was statistically higher in rural areas than in urban areas. Opposite to the study, the top 10 provinces and cities with greatest search volumes were characterized with a higher socioeconomic level and a sizable proportion of floating population. While 


\section{A}

TGI

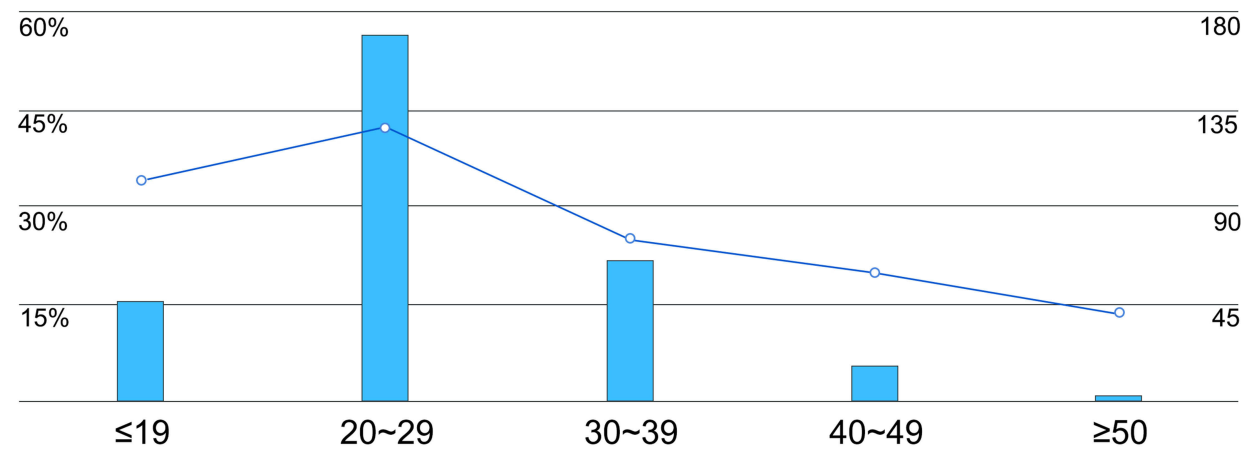

\section{B}

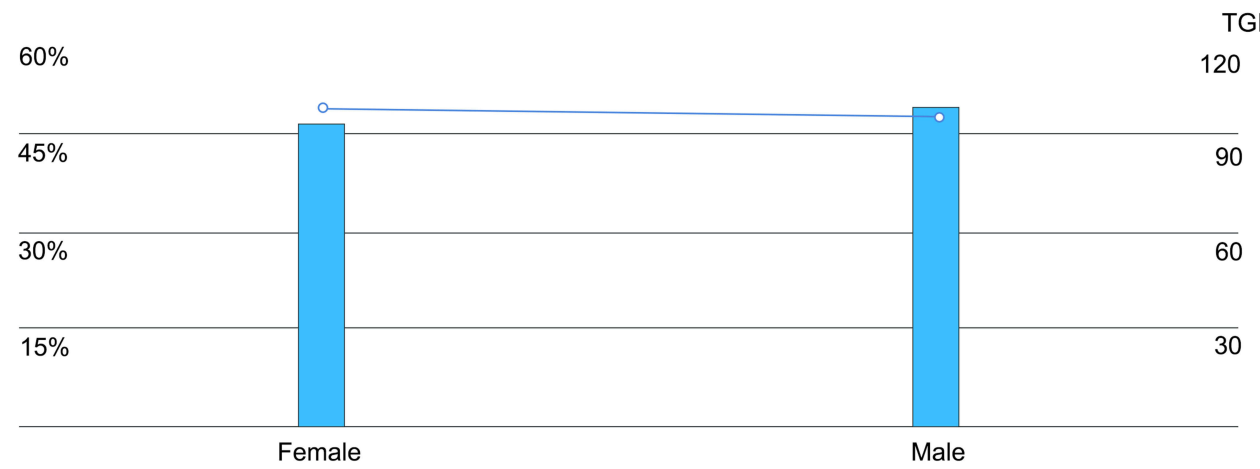

Figure 3 The demographic profiles by age $(\mathbf{A})$ and sex $(\mathbf{B})$.

this might demonstrate that the Baidu Index lacks adequate resolution to evaluate epidemiologic renal calculi trends at these levels, the obvious differences seen could also have resulted from a larger population, more perfect internet infrastructures, better medical conditions, and more public health awareness in economically developed areas. Furthermore, the kidney stones prevalence and distribution

Table I Categories of Top 10 Search Terms Related to Kidney Stones in the Baidu Index

\begin{tabular}{|l|l|l|}
\hline Rank & Category & Search Terms \\
\hline I & Diagnosis & "Symptoms of kidney stones" \\
3 & Treatment options & "The best treatment for kidney stones" \\
"Diet taboos for kidney stones patients" & "Kidney stones diet: 5 permissions and 3 \\
& & taboos" \\
4 & Etiology & "Kidney stones cause" \\
5 & Concomitant & "Renal cyst" \\
6 & diseases & "Hydronephrosis" \\
& General & "Stones" \\
& & "Ureteral stones" \\
& & "Urinary stones" \\
\hline
\end{tabular}

data from the study by Zeng et al used in the current study involved all urinary stones, not just kidney stones. Additionally, the cross-sectional survey of urolithiasis prevalence in China focused on permanent residents, while the Baidu Index could provide normalized search data emerged from the searches presented by all Baidu users, including Aboriginal and non-Aboriginal people. Hence, the reference

Table 2 Top 10 Rapidly Increasing Search Terms Related to Kidney Stones in the Baidu Index

\begin{tabular}{|l|l|}
\hline Rank & Search Term \\
\hline 1 & How to deal with kidney stones \\
2 & The best treatment for kidney stones \\
3 & Hydronephrosis \\
4 & Extracorporeal shock wave lithotripsy \\
5 & Urinary stones \\
6 & Severity of kidney stones \\
7 & Renal colic \\
8 & Indication of ESWL \\
9 & Urinary tract stones \\
10 & Kidney stones treatment \\
\hline
\end{tabular}




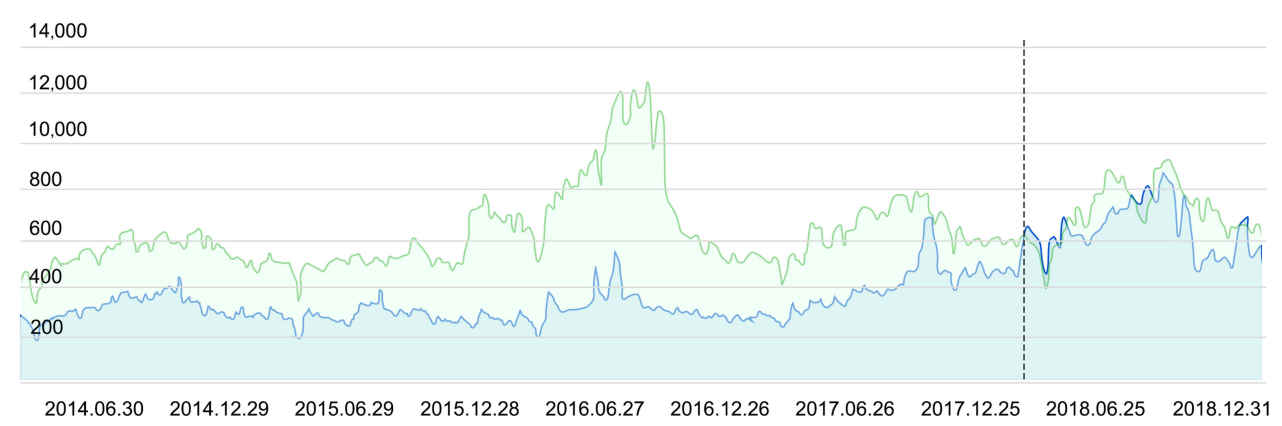

Figure 4 Baidu search volume index for "kidney stone surgery" (blue line) vs "extracorporeal shock wave lithotripsy” (green line). Marked dates January I2, 2018.

prevalence rates might not precisely represent the true incidence of kidney stones, measured by the Baidu Index. Internet users who search for healthcare information are $60 \%$ more likely to contact healthcare providers than those who do not use the internet. ${ }^{15}$ Also, online health information gathering can boost greater engagement in healthcare. ${ }^{16}$ In this sense, the "prevalence" represented by internet results may be more meaningful.

The vast majority of online search queries for "kidney stones" came from the male (50.18\%), aged 20-49 (83\%) groups. Despite their greater use of and access to the internet, the disproportionately high search volume coming from those groups related to "kidney stones" cannot be completely interpreted. ${ }^{4}$ Although the increased prevalence of renal calculi was positively association with age, ${ }^{1,13}$ considerable asymptomatic stone patients, especially the elderly in rural areas, were involved. They did not often seek treatment until sever symptoms presented due to less access to healthcare, lower income, a lack of medical insurance, and insufficient medical knowledge. ${ }^{17}$ Another explanation could be that the male, aged 20-49 group has a greater likelihood to suffer from symptomatic stones, and hence show a stronger need for kidney stones related information. Furthermore, the higher target group index (TGI) suggested a stronger interest in kidney stones related information among female netizens, despite having a lower prevalence of kidney stones corresponding to a smaller search volume. ${ }^{1,3,13}$

The data of the top search terms and rapidly increasing search terms generated from the Baidu Index can be used in educational and medical practice for healthcare providers. Changes in search term information over time can provide insights into patients' real-time concerns and priorities, going beyond the traditional quantitative and qualitative measures of disease surveillance. In this paper, results showed that searches related to the diagnosis, treatment, and diet control of nephrolithiasis ranked at the top 3. These results had nuances with the exiting research implemented in the United States via Google Trends, which suggested that searches related to the diagnosis, cause, and treatment ranked highly. With the help of these results, health educators and urologists, in their promotion and stone disease prevention activities, can better understand public needs through the analysis of demanding graph of kidney stones.

We found that the specific treatment modality "ESWL" was searched more frequently than the terminology "kidney stone surgery" over most of the past 5 years. This result was inconsistent with actual clinical rates using ESWL until a reversal occurred on January 12, 2018. Regarding trends in treatment modalities of renal calculi, recent studies found a considerable decline in ESWL with an increase in PCNL and URL procedures. ${ }^{18}$ ESWL search volume maintained a stable growth despite a considerable decline in actual clinical practice, perhaps because of individuals tending to favor the most cost-effective modality and a least invasive approach that required the least time and money. Besides, this might be attributed to excessive ESWL advertising on the internet that often exaggerated the treatment effects, public fear of surgery, and an increase in kidney stones prevalence. There was an obvious turning point in the search volume of kidney stones treatment modalities, occurring on January 12, 2018 and suggesting a possible new trend in surgical management of kidney stones consistent with actual practice. Interestingly, of the specific treatment modalities, "Percutaneous nephrolithotomy" and "ureteroscopy lithotripsy" were not included in the Baidu Index keywords database due to the low search volume despite a continued increasing trend in actual practice. This could suggest that Chinese internet users are searching ahead of visiting urologists, either upon "self-diagnosis" or diagnosis by a primary physician, lacking a thorough understanding of specific 
surgical treatment modalities. These findings prompt us to mobilize resources to strengthen initial patient education.

Utility of the Baidu Index is affected by some limitations. Most notably, this study was limited to the unique online search tool, Baidu, to Chinese terms only, and in China. Therefore, the data generated from other low-usage search engines was not assessed in our study. Furthermore, demographic data on the Baidu users was not available except for age and sex, eg, ethnicity, educational background, socioeconomic status, or other factors using Baidu. The Baidu Index may have sampling biases, as the profile of the internet users is significantly skewed to higher socioeconomic and better educated segments. Additionally, some important population segments might be left out, whose search queries do not contain the term "kidney stones". For example, a search query containing "Urinary stones" was not collected in our analysis. Our study was thus likely to underrate the target population with kidney stones. Besides, the implementer's motivation for conducting online searches was not available, because a patient, researcher, healthcare provider possibly all contributed to the search volume in kidney stones. It remains to be studied how this will confuse the use of the Baidu Index for estimating the prevalence status of kidney stones. Another weakness of using the Baidu Index is that the true search volume is not publicly available and subject to abrupt increases with considerable media coverage irrelevant for the real incidence of disease.

Despite these limitations, this study demonstrated that a growing number of individuals regularly utilize online search engine to consult information related to "kidney stones" and treatment options. Internet search trend data is a valuable source for monitoring kidney stones-related information-seeking behavior and can be used as an exploratory tool for better understanding of patients' features and priorities. It may help provide targeted health information services online, scientific evidence for kidney stone disease control, and prevention strategies in China. Future studies will be conducted to assess the reliability and stability of utilizing internet big data as a surveillance method.

\section{Conclusion}

The Baidu Index can be utilized to track Chinese online behavior and interest in health topics. It may help improve our understanding of disease incidence and population interest, direct resource utilization, and patient education. With increased kidney stones prevalence during the past decades, online search rates have maintained sustained growth. The geographic and demographic characteristics in urolithiasis seem to be presented via online search data analysis to some extent. A faster-growing search volume related to "kidney stone surgery" showed a possible changing trend in the treatment of kidney stones.

\section{Disclosure}

All authors declare no conflicts of interest in this study.

\section{References}

1. Zeng G, Mai Z, Xia S, et al. Prevalence of kidney stones in China: an ultrasonography based cross-sectional study. BJU Int. 2017;120 (1):109-116. doi:10.1111/bju.13828

2. Trinchieri A, Coppi F, Montanari E, Del Nero A, Zanetti G, Pisani E. Increase in the prevalence of symptomatic upper urinary tract stones during the last ten years. Eur Urol. 2000;37(1):23-25. doi:10.1159/ 000020094

3. Scales CD Jr., Smith AC, Hanley JM, Saigal CS. Prevalence of kidney stones in the United States. Eur Urol. 2012;62(1):160-165. doi:10.1016/j.eururo.2012.03.052

4. China Internet Network Information Center (CNNIC). The 44th China statistical report on internet development; 2019. Available from:http://www.cnnic.net.cn/hlwfzyj/. Accessed Aug 30, 2019.

5. China Internet Network Information Center (CNNIC). China statistical report on search engine; 2019. Available from: http://www. cnnic.net.cn/hlwfzyj/. Accessed Oct 25, 2019.

6. Nuti SV, Wayda B, Ranasinghe I, et al. The use of google trends in health care research: a systematic review. PLoS One. 2014;9(10): e109583. doi:10.1371/journal.pone.0109583

7. Eysenbach G. Infodemiology and infoveillance: framework for an emerging set of public health informatics methods to analyze search, communication and publication behavior on the Internet. $J \mathrm{Med}$ Internet Res. 2009;11(1):e11. doi:10.2196/jmir.1157

8. Chen S. Big data applications and practices of Baidu. Big Data Res 2015;(1):97-107.

9. Breyer BN, Sen S, Aaronson DS, Stoller ML, Erickson BA, Eisenberg ML. Use of google insights for search to track seasonal and geographic kidney stone incidence in the United States. Urology. 2011;78(2):267-271. doi:10.1016/j.urology.2011.01.010

10. Willard SD, Nguyen MM. Internet search trends analysis tools can provide real-time data on kidney stone disease in the United States. Urology. 2013;81(1):37-42. doi:10.1016/j.urology.2011.04.024

11. Dreher PC, Tong C, Ghiraldi E, Friedlander JI. Use of google trends to track online behavior and interest in kidney stone surgery. Urology. 2018;121:74-78. doi:10.1016/j.urology.2018.05.040

12. Brikowski TH, Lotan Y, Pearle MS. Climate-related increase in the prevalence of urolithiasis in the United States. Proc Natl Acad Sci US A. 2008;105(28):9841-9846. doi:10.1073/pnas.0709652105

13. Stamatelou KK, Francis ME, Jones CA, Nyberg LM Jr, Curhan GC. Time trends in reported prevalence of kidney stones in the United States: 1976-1994. Kidney Int. 2003;63(5):1817-1823. doi:10.1046/ j.1523-1755.2003.00917.x

14. Atan L, Andreoni C, Ortiz V, et al. High kidney stone risk in men working in steel industry at hot temperatures. Urology. 2005;65 (5):858-861. doi:10.1016/j.urology.2004.11.048

15. Ybarra ML, Suman M. Help seeking behavior and the internet: a national survey. Int J Med Inform. 2006;75(1):29-41. doi:10.1016/j.ijmedinf. 2005.07.029

16. Iverson SA, Howard KB, Penney BK. Impact of internet use on health-related behaviors and the patient-physician relationship: a survey-based study and review. J Am Osteopath Assoc. 2008;108 (12):699-711. 
17. National Bureau of Statistics of China. All the basic information and points balance urban and rural residents. Available from: http://data. stats.gov.cn/easyquery.htm?cn=C01. Accessed Dec 30, 2018.
18. Chung KJ, Kim JH, Min GE, et al. Changing trends in the treatment of nephrolithiasis in the real world. J Endourol. 2019;33(3):248-253. doi:10.1089/end.2018.0667

\section{Publish your work in this journal}

Risk Management and Healthcare Policy is an international, peerreviewed, open access journal focusing on all aspects of public health, policy, and preventative measures to promote good health and improve morbidity and mortality in the population. The journal welcomes submitted papers covering original research, basic science, clinical \& epidemiological studies, reviews and evaluations, guidelines, expert opinion and commentary, case reports and extended reports. The manuscript management system is completely online and includes a very quick and fair peer-review system, which is all easy to use. Visit http://www.dovepress.com/testimonials.php to read real quotes from published authors. 